\title{
An Effective Feature Extraction Approach for Iris Recognition System
}

\author{
Krishna Devi, Preeti Gupta, Diksha Grover and Anaahat Dhindsa \\ Department of Electronics and Communication Engineering, UIET, Panjab University, \\ Chandigarh -160014,Panjab, India; Krishnadevi888@gmail.com, Preeti.uiet@gmail.com, \\ d.grover1092@gmail.com, Anaahat.dhindsa85@gmail.com
}

\begin{abstract}
Background/Objectives: Iris recognition system refers to a system used for identifying different iris texture patterns for different applications. The research is aimed at developing a system that improves the efficiency of the iris recognition system and make it more reliable and robust. Methods: To this end, we have developed a system based on compound local binary pattern technique. Compound local binary technique is a spatial domain technique, which assign a $2 \mathrm{P}$ bit code to central pixel based on the local neighbourhood comprising of $\mathrm{P}$ neighbours. The operator takes into consideration both the sign and magnitude information of the central and corresponding neighbour grey values. The unique and abundant features extracted through Compound Local Binary Pattern (CLBP) operator act as input to the neural network classifier. Findings: The system has been tested over 50 eye images taken from CASIA database. Iris recognition system based on Compound local binary pattern technique along with neural network used as classifier improves the accuracy of system in comparison to existing feature extraction approaches. In this proposed research, recognition rate achieved is $96 \%$.
\end{abstract}

Keywords: Compound Local Binary Pattern (CLBP), Feature Extraction, Iris Localisation, Iris Recognition, Neural Network

\section{Introduction}

At whatever point individuals sign on to PCs, get to an ATM, go through high security territories, enter air terminal security, they have to prove their identities. Commonly individuals make utilization of passwords and ID cards to demonstrate that they are who they claim to be. However passwords could be overlooked, ID cards could be stolen or lost. Along these lines an enormous change has been done to enhance this technique for identification 1 . Biometric deals with automated method of recognizing an individual on the basis of their physiological and behavioural characteristics such as face, fingerprint, hand-geometry, retinal, DNA, signature etc. unlike other biometric system iris proves to be more reliable, exhibit long term stability, highly secure method of recognizing individuals ${ }^{2}$. Moreover, the left and right eye of person has unique features. The iris pattern is unique in case of twin's also ${ }^{3}$.
This paper is composed as takes after. Segment II surveys essential foundation ideas in iris life structures. Segment III represents description about database. Segment IV exhibited the work which has already been done over iris acknowledgment framework. Section V-VIII audit essential result identified with one of the four modules of an iris biometrics framework: Iris localization, Iris normalization and Feature extraction.

\section{Iris Anatomy}

Iris is colored ring of tissue around the pupil region. The light enters into eye through this pupil region. There are two muscles sphincter and dilator, which basically controls the size of iris. The iris region is surrounded by a white region of connective tissues and blood vessels, known as sclera. The iris region has typical pattern of furrows, ridges collarette and pigment $\operatorname{spot}^{4}$ as shown in

${ }^{*}$ Author for correspondence 
Figure 1. The rich texture pattern of iris is revealed in near infrared illumination 2 .

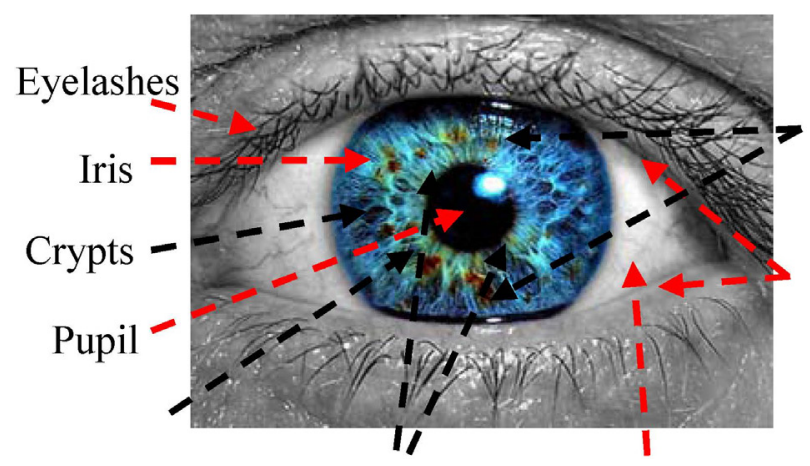

Figure 1. Iris anatomy $\underline{5}^{\underline{5}}$

\section{Database}

A number of databases are available such as CASIA, IIT Delhi, MMU, UBIRIS etc. CASIA database is used in this research work. The Chinese academy of sciences-institute of automation contains 756 grey scale eye images with 108 unique images and 7 different images of each unique eye. The images are shown below in Figure 2. The images are captured using specialized digital optics and under certain constraints.
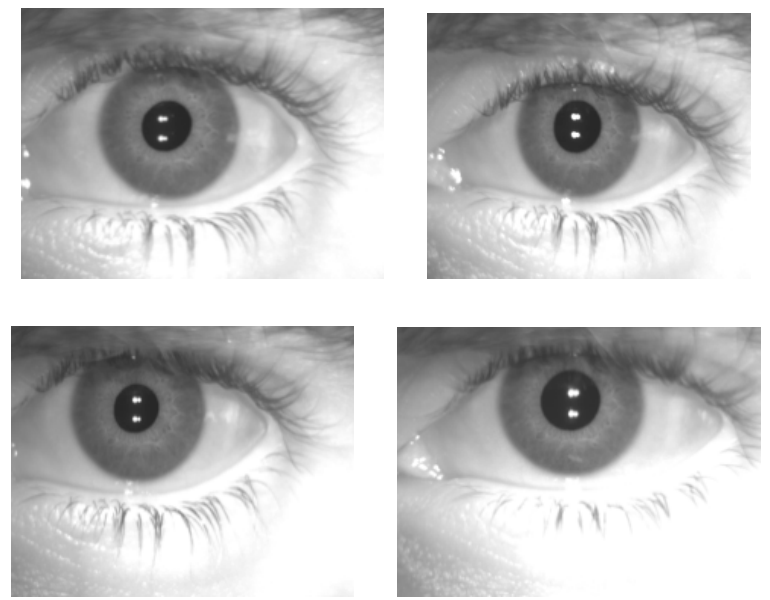

Figure 2. Grey scale eye images from CASIA database

\section{Related Work}

$\mathrm{In}^{6}$ this research work Linear Binary pattern technique is used for extracting features. Linear vector quantization classification approach uses this feature vector for classifying individuals. Matching is performed using
Hamming distance. The feature extraction technique is applied on CASIA database. $\operatorname{In}^{\underline{7}}$ proposed work a new feature extraction approach known as Neighbourhood binary pattern. In this unique feature extraction approach the central pixel value is compared with the neighbours of central pixel and encoding it by 1 if it has a larger value in comparison to central pixel otherwise assigning a 0 value. The obtained code is converted into binary form. This serve as feature descriptor for the respective image. The proposed method achieves an accuracy rate of $75 \%$. The technique has been applied over CASIA database. $\operatorname{In}^{8}$ this paper presented linear binary pattern technique for extracting texture features is used. K-nn and NB classifiers are used for classification. Hamming distance is used for matching. The accuracy achieved through K-nn classifier is more in comparison to NB classifier and other conventional methods. $\mathrm{In}^{9}$ order to improve the accuracy and performance of face recognition method, introduced a new and powerful feature extraction approach i.e. Compound Local Binary Pattern (CLBP). The technique has been applied over ORL database. Fusion of both spatial domain and transform domain techniques is done. A high recognition rate is achieved through this approach. In ${ }^{10}$ this proposed work constructed facial feature descriptor using compound local binary pattern technique. This technique overcomes the limitation of linear binary pattern technique. The technique has been applied over Japanese female facial expression and cohn-kanade database. Support vector machine is used for classification. This paper11 presented linear binary pattern technique for extracting features from an iris image. For classification neural network is used. The accuracy rate achieved through this approach is $93.5 \%$. $\mathrm{In}^{12}$ this proposed work features are extracted using linear binary pattern technique. The matching is done by using SVM classifier and hamming distance approach and comparison has been done using these two approaches. A high level of accuracy is achieved by using classifiers.

\section{Proposed Methodology}

This paper mainly focuses on extracting feature form an image taken from CASIA database. Initially the image is acquired with the help of digital camera. Circular Hough transform is applied over the acquired image. Canny edge detector is used for detecting lines and edges within image. The iris and pupil co-ordinates and radi- 
uses are calculated by means of circular Hough transform approach. The segmented images obtained after segmentation are of different sizes. Normalisation is done for converting Cartesian co-ordinates into polar coordinates. Compound local binary pattern technique is applied over the normalised image for extracting features. The obtained feature vector is applied to neural network classifier. The block diagram of iris recognition system is shown in Figure 3:

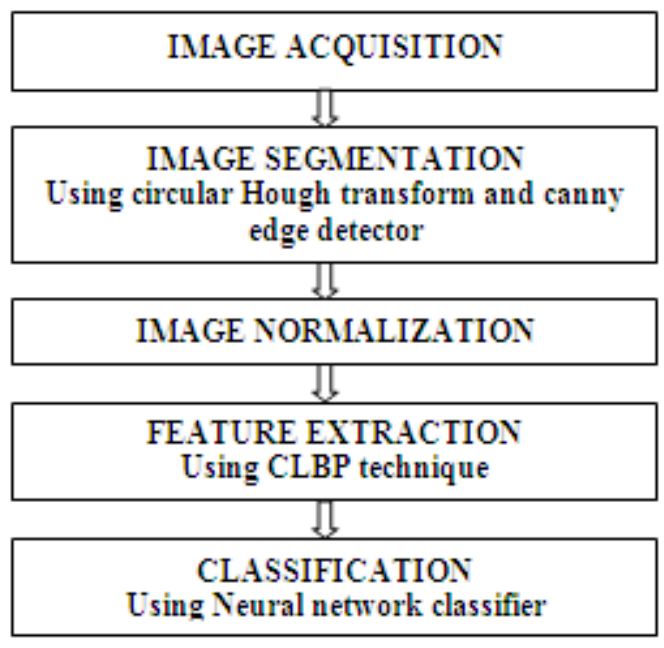

Figure 3. Block diagram of iris recognition system

\section{Steps Involved in Iris Recognition System}

\subsection{Image Acquisition}

This step involves acquiring an image in near infrared illumination with the help of high resolution digital camera. Digital camera uses an image sensor. The digital representation of image saves memory space.

\subsection{Image Localisation}

The image acquired with the help of digital camera is further pre-processed to detect the iris region. The canny edge detector is applied in order to detect the lines and edges within an image. The first step is to locate iris and pupil regions and their co-ordinates. Circular Hough transform is applied in order to detect the iris and papillary region and evaluate their radius ${ }^{\frac{13}{3}}$.

\subsection{Image Normalisation}

The images obtained after localisation and pre-processing are of varying sizes. This deformation needs to be compensated for accurate analysis of texture pattern of an image. This module basically converts Cartesian co-ordinates into polar co-ordinates.

\subsection{Feature Extraction using CLBP}

It is an extended version of linear binary pattern texture operator. This technique has never been applied over iris texture pattern before. This technique is highly reliable, secure and achieve high recognition rate in case of facial recognition systems. Linear binary pattern texture operator rejected the magnitude information of the difference between centre and neighbouring pixels. This technique produces inconsistent codes. In order to overcome the shortcomings of linear binary pattern technique and to improve the performance of biometric system, we proposed CLBP technique. The technique employs a $2 \mathrm{P}$ bit code to central pixel on the basis of grey values comprising of $\mathrm{P}$ neighbours. The first bit represents the sign and second bit represents magnitude of the difference between the centre and the corresponding neighbour values. $X_{\text {avg }}$ is the average magnitude of the difference between the centre and the neighbouring grey values. This technique assign 1 bit if the magnitude of difference between the central pixel and neighbours is more in comparison to $X_{\text {avg } 10}$.

$$
Z\left(i_{p}, i_{c}\right)=\left\{\begin{array}{ccc}
00 & i_{p}-i_{c}<0 & \left(\left|i_{p}-i_{c}\right| \leq X_{\text {avg }}\right) \\
01 & i_{p}-i_{c}<0 & \left(\left|i_{p}-i_{c}\right|>X_{\text {avg }}\right) \\
10 & i_{p}-i_{c} \geq 0 & \left(\left|i_{p}-i_{c}\right| \leq X_{\text {avg }}\right) \\
11 & \text { otherwise }
\end{array}\right.
$$

$i_{p}$ represents the grey values of neighbouring pixels and ${ }^{i} c$ represents the grey value of central pixel. The clbp operator works as shown in Table 1 below:

The code for central pixel C is 1010101010111111.

CLBP technique is basically a local texture feature extraction technique. It takes into consideration the spatial relationship between pixel values. Histograms are plotted for each CLBP image and encoded. 
Table 1. CLBP technique

\begin{tabular}{|l|l|l|}
\hline 61 & 61 & 110 \\
\hline 60 & 60 & 110 \\
\hline 60 & 61 & 109 \\
\hline
\end{tabular}

\begin{tabular}{|l|l|l|}
\multicolumn{3}{|c}{ 少 } \\
\hline 10 & 10 & 11 \\
\hline 10 & $\mathrm{C}$ & 11 \\
\hline 10 & 10 & 11 \\
\hline
\end{tabular}

\section{Classification using Neural Network Classifier}

Neural network approach is used for classification. Once the features are extracted using CLBP technique, an iris normalised images has transformed into feature vector. This feature descriptor serves as input to the classifier. A subset of 50 images of 12 persons has been taken from the CASIA database. In this proposed work 40 images from database are used for training and 15 for testing purpose.

\section{Results}

In order to experiment the proposed feature extraction technique and to compute the recognition rate CASIA database has been considered. The following Figures 4-9 shows the different steps involved in iris recognition system and the output results obtained after each step.

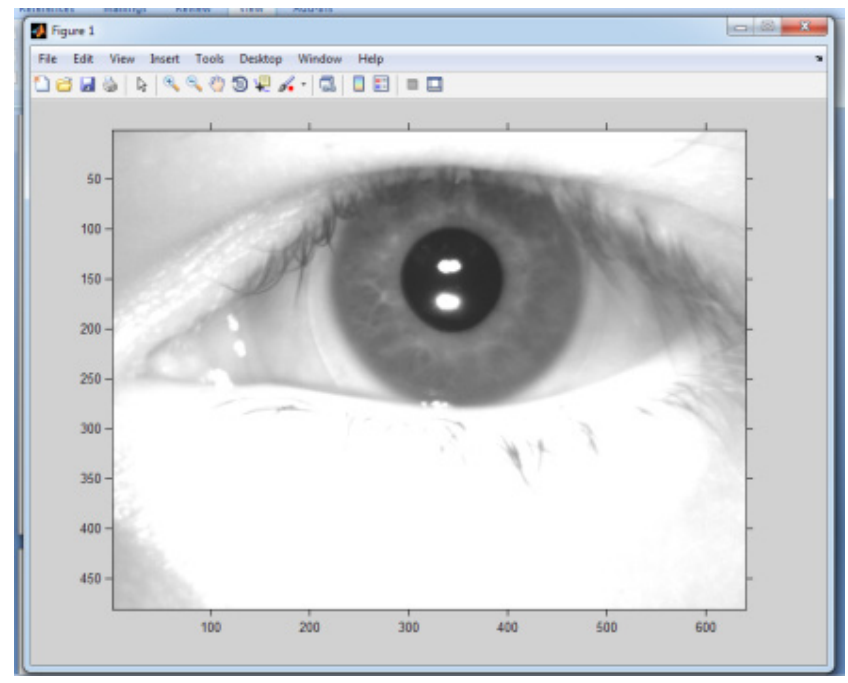

Figure 4. Original eye image.

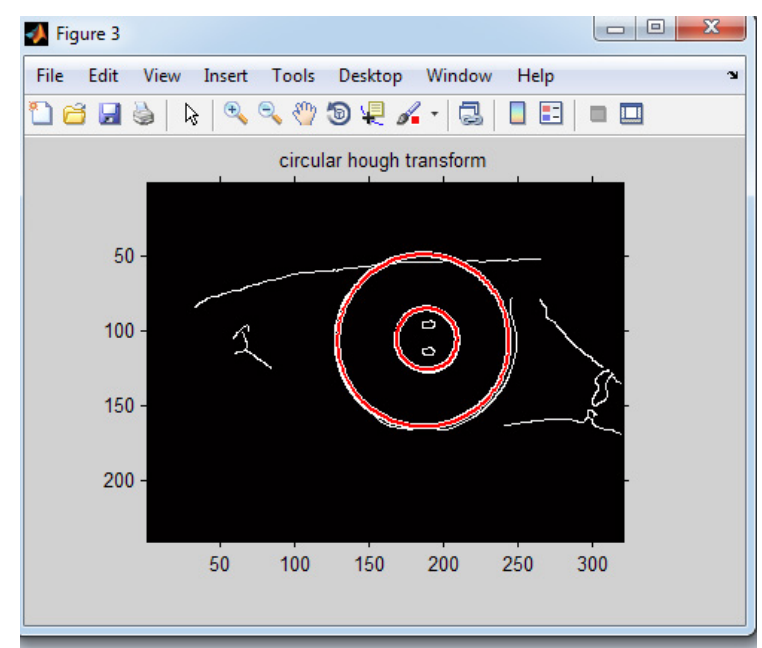

Figure 5. Eye image after applying circular Hough transform.

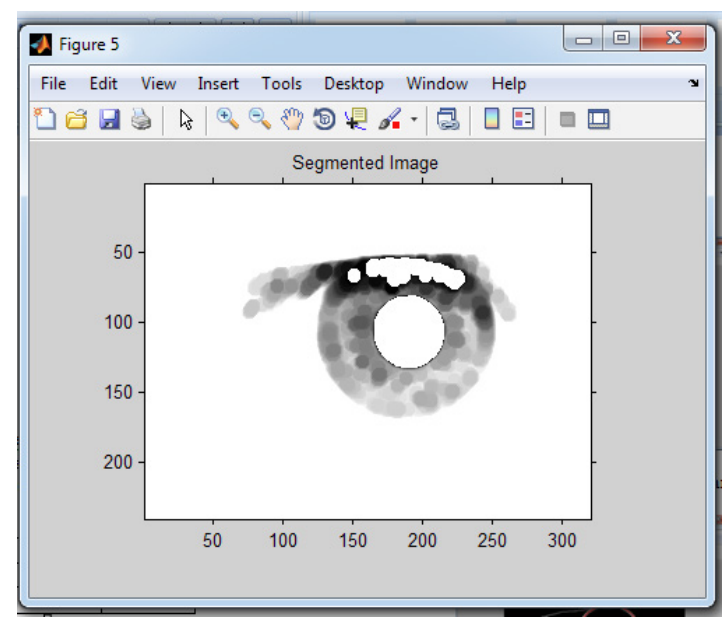

Figure 6. Segmented image.

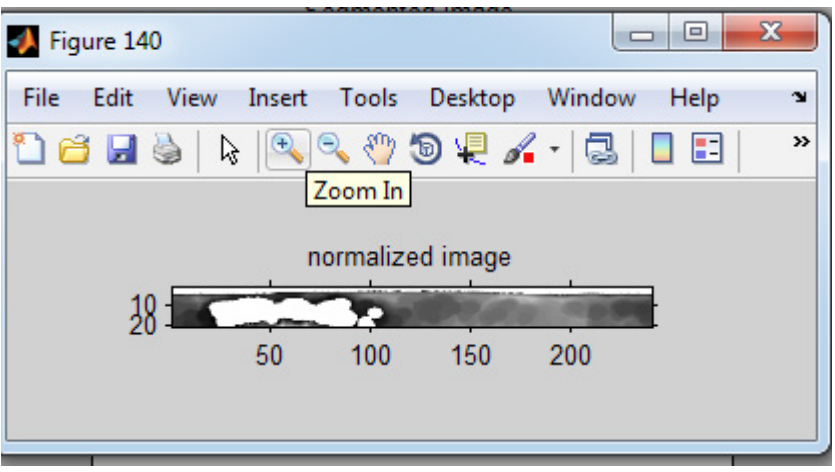

Figure 7. Normalised image.

These steps are computed for the whole dataset consisting of 50 images and after that recognition rate is calculated. 


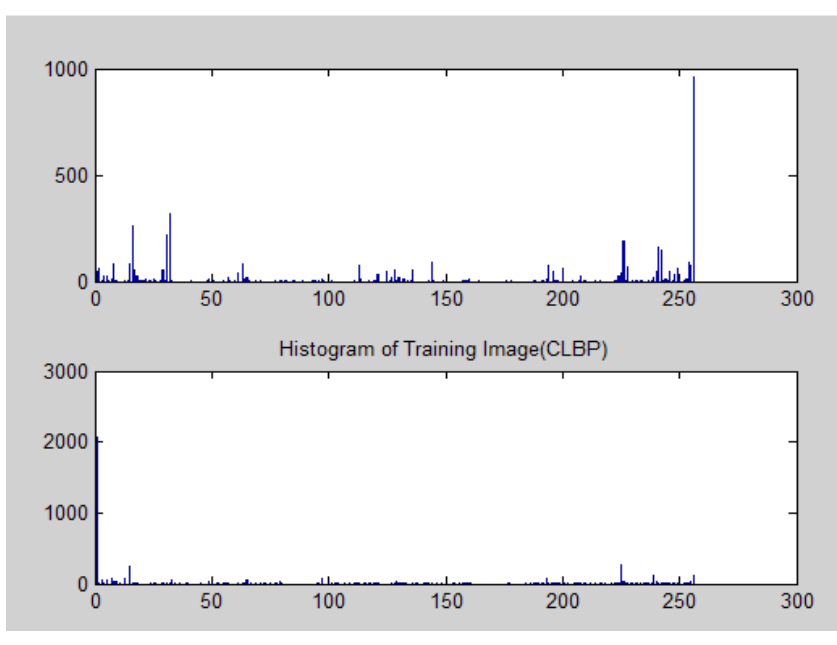

Figure 8. Histogram of CLBP image.

The recognition rate achieved using CLBP technique after training using images from CASIA database (Table 2).

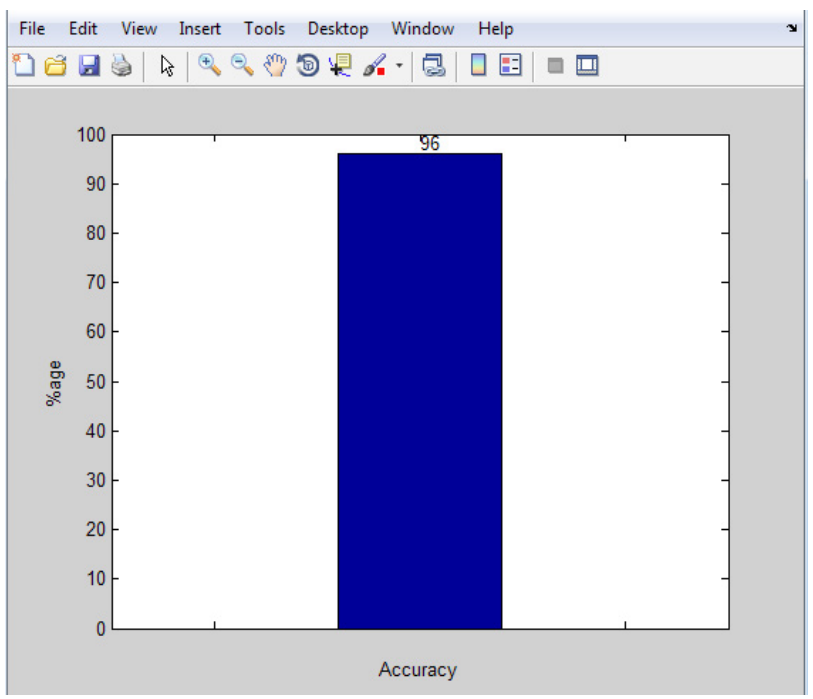

Figure 9. Recognition rate

Table 2. Comparison between two systems

\begin{tabular}{|l|l|l|}
\hline Feature extraction & Classifier & Recognition rate \\
\hline CLBP & Neural Network & $96 \%$ \\
\hline LBP & Neural network & $93.5 \%$ \\
\hline
\end{tabular}

\section{Conclusion}

The iris recognition is an effective and highly reliable physiological biometric trait for identifying an individual. In this paper iris recognition uses spatial domain technique known as compound local binary pattern technique. The CLBP operator provides an effective and efficient approach for iris feature representation with high discriminative ability. CLBP provides better recognition rate in comparison to LBP technique.

\section{References}

1. Bowyer KW, Hollingsworth K, Flynn PJ. Image Understanding for Iris Biometric: A Survey, Computer Vision and Image Understanding. 2007; 110(2):281-307.

2. Daughman J. How Iris Recognition Works, IEEE Transaction on Circuit and System for Video Technology. 2004; 14:21-30.

3. Rakesh T, Khogare MG. Survey of Biometric Recognition System for Iris, International Journal of Emerging Technology and Advanced Engineering. 2012; 2(6).

4. Tallapragada VVS, Rajan EG. Iris Recognition Based on Combined Feature of GLCM and Wavelet Transform, First International Conference on Integrated Intelligent Computing, 2010, p.205-10.

5. Aydi W, Fadhel N, Masmoudi N, Kamoun L. A Robust Feature Extraction Method Based on Monogenic Filter for Iris Recognition System. IEEE. 2014.

6. Sharma A, Gupta R. Iris Recognition Based Learning Vector Quantization and Local Binary Pattern on Iris Matching, International Journal of Technical Research and Application. 2015; 3(5):7-14.

7. Hamouchene I. A New Texture Analysis Approach for Iris Recognition, AASRI Conference on Circuit and Signal Processing. 2014; 9:2-7.

8. Sarode NS, Patil AM. Iris Recognition using LBP and Classifier - Knn and NB, International Journal of Science and Research. 2015; 4(1).

9. Patwardhan T, Byalal RM. Implementation of Fusing Based Compound Local Binary Pattern Algorithm for Face Recognition, International Journal of Advanced Research in Computer Engineering and Technology. 2016 May; 5(5).

10. Ahmed F, Bari H, Hossain E. Person Independent Facial Expression Recognition Based on Compound Local Binary Pattern, International Arab Journal of Information and Technology. 2014 Mar; 11(2).

11. Hajari K. Improving Iris Recognition Performance using Local Binary Pattern and Combined RBFNN, International Journal of Engineering and Technology. 2015 Apr; 4(4).

12. Saminathan K, Chakravarthy T, Devi C. Comparative Study on Biometric Recognition Based on Hamming Distance and Multi Block Local Binary Pattern, Indian Journal of Science and Technology. 2015 Jun; 8(11).

13. Rai H, Yadav A. Iris Recognition using Combined Support Vector Machine and Hamming Distance, Expert Systems with Applications. 2014. 